\title{
FATORES DETERMINANTES DA PROBABILIDADE DE DIVULGAÇÃO VOLUNTÁRIA DE INFORMAÇÕES FINANCEIRAS DAS EMPRESAS ${ }^{1}$
}

\section{DETERMINANTS OF THE PROBABILITY OF VOLUNTARY DISCLOSURE OF THE COMPA- NY'S FINANCIAL INFORMATION}

\author{
Samuel Lyncon Leandro de Lima \\ Mestre em Ciências Contábeis (UNIOESTE) \\ Universidade Estadual do Oeste do Paraná \\ samuellynconleandro@gmail.com \\ Maria da Piedade Araújo \\ Doutora em Economia (USP) \\ Universidade Estadual do Oeste do Paraná \\ madadepi@gmail.com \\ Fernando Dal Ri Murcia \\ Doutor em Ciências Contábeis (USP) \\ Universidade de São Paulo \\ murcia@usp.br
}

\section{RESUMO}

Objetivo: O propósito da pesquisa foi analisar os fatores determinantes que influenciaram a probabilidade das companhias não financeiras de capital aberto, listadas na Brasil Bolsa Balcão a realizarem a prática de disclosure de informações financeiras voluntárias no ano de 2016.

Fundamento: A divulgação voluntária de informações consiste em uma temática com papel fundamental na contabilidade, pois discute aspectos associados à transparência de informações das organizações.

Método: O diferencial metodológico proposto nessa pesquisa foi construir as variáveis dependentes, com o auxílio de uma métrica qualitativa. Foram realizadas coletas para levantamento sobre as informações financeiras publicadas voluntariamente em 267 companhias e, consequentemente, analisadas em profundidade, objetivando construir as variáveis dependentes dos modelos de regressão. Já as variáveis explicativas, foram modeladas com base em estudos anteriores, relativas à rentabilidade, ao endividamento, ao tamanho, a governança corporativa e a auditoria. Por conseguinte, para a análise dos dados, empregou-se a modelagem de regressão logit. Além disso, no intuito de analisar a validade preditiva dos modelos estimados, empregou-se o teste de HosmerLemeshow.

\footnotetext{
${ }^{1}$ Artigo recebido em: 12/03/2020. Revisado por pares em: 04/09/2020. Reformulado em: 13/09/2020. Recomendado para publicação: 04/09/2020 por Marco Aurélio dos Santos (Editor Adjunto). Publicado em: 11/02/2021. Organização responsável pelo periódico: UFPB
} 
Resultados: Os principais resultados consistem nas evidências da governança corporativa e da auditoria influenciarem significativamente as práticas de disclosure de informações financeiras das empresas. Dessa maneira, os resultados evidenciaram que as empresas listadas no Novo Mercado não só têm alta probabilidade de publicarem voluntariamente, como divulgam de maneira mais detalhada, mostrando-se mais transparência.

Contribuições: Essa pesquisa contribui para a literatura da divulgação de informações, proporcionando evidências sobre a relevância do nível de governança corporativa e da auditoria independente na maximização da probabilidade da divulgação voluntária.

Palavras-chave: Teoria da divulgação voluntária. Governança corporativa. Contabilidade. Auditoria. Desempenho econômico e financeiro.

\section{ABSTRACT}

Objective: The purpose of the study was to analyze the determinants that influenced the probability of nonfinancial companies listed in the Brasil Bolsa Balcão to practice the disclosure of voluntary financial information in 2016.

Background: The voluntary disclosure of financial information consists of a theme with a fundamental role in accounting, since it discusses aspects related to the transparency of information of the organizations.

Method: The methodological differential proposed in this research was to build the dependents variables, with the aid of a qualitative metric. It was collected the data of financial information voluntarily published in 267 companies and, consequently, analyzed in depth, aiming to build the dependents variables on the regression models. The explanatory variables, on the other hand, were modeled based on previous studies, related to profitability, indebtedness, size, corporate governance and auditing. Therefore, for data analysis, logit regression modeling was used. In addition, in order to analyze the predictive validity of the estimated models, the Hosmer-Lemeshow test was used.

Results: The main results consist of the significant influence evidence of corporate governance and audit on the companies' financial information disclosure practices. In this way, the results showed that the companies listed on the New Market not only more disclose voluntarily, but also disclose in more detail, showing more transparency.

Contributions: This research contributes to the information disclosure literature, providing evidence about the relevance of the level of corporate governance and independent auditing in maximizing the probability of voluntary disclosure.

Keywords: Theory of voluntary disclosure. Corporate governance. Accounting. Audit. Economic and financial performance.

\section{INTRODUÇÃO}

A função-objetivo das empresas direcionam os gestores à tomada de decisões (Gama Boaventura, Cardoso, Silva, \& Silva, 2009). Nessa perspectiva, atingir o objetivo da organização e evidenciar aos interessados as informações da empresa, constitui-se em uma tarefa crítica. Isso se deve, em razão da expectativa dos interessados em um ambiente formado pela livre concorrência, percebendo as organizações como um dos fatores mais relevantes para progresso econômico (Madden, 2017).

A expectativa dos participantes do mercado de capital, segundo Asay, Elliott e Rennekamp (2016) ao acessarem as informações das empresas e, se perceberem superficialidade no conteúdo divulgado, sentem-se menos confortáveis para avaliar a respectiva organização, adotando uma postura cautelosa quanto ao seu desempenho futuro. 
Destaca-se nesse contexto, a contabilidade, que possui um aspecto prático histórico relevante, caracterizado como instrumento de controle da organização e de seus usuários, da prestação de contas desses agentes e, fornecimento de informações direcionadas para tomada de decisão por parte dos diversos agentes interessados pelas informações contábeis (Iudícibus, Martins, \& Carvalho, 2005).

Em se tratando de informações contábeis, emergem os estudos voltados à compreensão dos aspectos relacionados à divulgação voluntária, que segundo Verrecchia (2001) consiste em uma temática com papel central na contabilidade. A discussão em torno da Teoria da Divulgação possui uma significativa relação com os aspectos voltados à redução da assimetria informacional, iniciadas com os trabalhos tradicionais encontrados na literatura de Verrecchia (1983) e Dye (1985).

Essa discussão consiste no pressuposto de que os gestores providos de informações particulares sobre as organizações, cuja divulgação não está classificada em caráter obrigatório, decide evidenciar determinadas informações aos investidores e interessados, reduzindo consequentemente a assimetria de informações entre os agentes participantes da organização (Verrecchia, 1983).

Destaca-se, de acordo com Alves, Canadas e Rodrigues (2015), ser crescente a importância sobre o tratamento da divulgação de informações, como um mecanismo de transmitir segurança aos investidores. Aliado a isso, a prática da divulgação está intimamente associada ao interesse dos gestores em promover a transparência entre a organização e o mercado (Alves et al., 2015).

Diante disso, a pesquisa possui uma abordagem direcionada à divulgação de informações voluntárias de natureza financeira. Assim, foram verificados diversos estudos nacionais que tratam à temática, como um importante campo do conhecimento contábil a ser explorado, haja vista a significativa relevância oportunizada pela transparência de informações particulares das empresas (Mendes-da-Silva \& Magalhães Filho, 2005; Luz, Camargo, \& Lima, 2006; Salotti \& Yamamoto, 2008; Murcia, 2009; Murcia \& Santos, 2009b; Cruz \& Lima, 2010; Silva, Alberton, \& Vicente, 2013; Rufino \& Monte, 2014; Sousa, Silva, Ribeiro, \& Weffort, 2014; Forte, Perez, Bessa, \& Oliveira, 2015; Forte, Santos Neto, Nobre, Nobre, \& Queiroz, 2016; Mota \& Pinto, 2017).

Além desses estudos nacionais, verificou-se que a divulgação de informações também possui uma relevância significativa no cenário internacional, pela qual pode ser constatada em estudos recentes de diversos países (Alfaraih \& Alanezi, 2011; Kateb, 2012; Al-Janadi, Rahman, \& Omar, 2013; Barac, Granic, \& Vuko, 2014; Adam, Mukhtaruddin, \& Putri, 2014; Thoresson \& Niléhn, 2014; Birjandi, 2015; Hieu \& Lan, 2015; Zaini, Sharma, \& Samkin, 2017; Dolinšek \& Lutar-Skerbinjek, 2018; Nguyen, Nguyen, \& Nguyen, 2020).

Considerando a relevância do tema, e as abordagens propostas nessas pesquisas anteriores, verificou-se uma lacuna de pesquisa, pois não se encontrou trabalhos que avaliassem a variável dependente (publicar ou não informações voluntárias) qualitativamente. Dessa maneira, constatou-se a oportunidade de propor uma alternativa de mensuração da divulgação de informações contábeis, com propósito de avançar as discussões para além de divulgar ou não, introduzindo um enfoque de qualificação do conteúdo divulgado.

Além disso, compreendeu-se a lacuna de analisar os determinantes da divulgação voluntária de informações financeiras, verificados principalmente a partir do estudo de Murcia (2009), sob a abordagem de mensuração da informação publicada voluntariamente proposta nessa pesquisa. Sendo assim, os determinantes da divulgação voluntária, como a rentabilidade, o endividamento, o tamanho, a governança e a auditoria foram analisados nessa pesquisa.

Isto posto, a pesquisa foi norteada pela seguinte questão: qual a probabilidade de as variáveis rentabilidade, endividamento, tamanho, governança e ser auditada pelas Big Four, influenciarem as práticas de disclosure voluntário de informações financeiras das companhias brasileiras não financeiras de capital aberto listadas na Brasil Bolsa Balcão no ano de 2016? 
Espera-se que a discussão proposta nessa pesquisa possa contribuir com os participantes do mercado de capital, na medida em que é por meio das informações divulgadas que os investidores efetuarão análises para compreender o desempenho da organização e, consequentemente, realizar ou não o investimento de seus recursos nessa empresa. Ademais, acredita-se que o estudo possa contribuir com as discussões futuras, uma vez que com a introdução de variáveis de categorias, possibilitará estimar a probabilidade da divulgação de informações voluntárias com informações qualitativas, uma das restrições apresentadas nos estudos anteriores.

Este estudo, além dessa introdução, apresenta: o Referencial Teórico, sobre a Teoria da Divulgação Voluntária, estudos empíricos anteriores e desenvolvimento das hipóteses; a Metodologia, pela qual são apresentados os procedimentos metodológicos, a base de dados, a amostra e os modelos estimados; a Análise dos Resultados, com os testes de robustez dos modelos e, por fim; as Considerações Finais.

\section{BASE TEÓRICA}

\subsection{Teoria da Divulgação}

Estudos como o de Verrecchia (1983) buscam estudar a divulgação voluntária de informações, sobre a abordagem de identificar os fatores determinantes sobre a prática da divulgação ou não de informações particulares das organizações.

Acredita-se, segundo Dye (1985), que os gestores detenham conhecimento sobre as informações das organizações pelas quais são responsáveis pela gestão, por exemplo, aquelas relacionadas aos ganhos anuais e, na medida em que forem evidenciadas, causam um impacto direto no valor das ações, sem afetar, no entanto, a distribuição de lucros futuros dessas organizações. Assim como Verrecchia (1983), em sua pesquisa, Dye (1985) buscou identificar os aspectos relacionados ao comportamento dos investidores em divulgar ou não as informações das empresas pelos quais detêm conhecimento.

Diante disso, a discussão sobre a retenção de informações particulares de uma organização, segundo Dye (1985), é caracterizada como uma inquietação, pela expectativa do impacto da informação, visto que se os investidores identificarem que os gestores possuem determinadas informações sobre a empresa, é provável que os gestores realizem a divulgação. É importante salientar que os investidores possuem uma inquietação relacionada à liquidez da organização, por esse motivo, demandam informações que estejam relacionadas ao desempenho da empresa (Diamond \& Verrecchia, 1991).

No tratamento dado à divulgação de informações, em uma pesquisa de levantamento realizada na temática, por meio dos estudos anteriores, com relação à divulgação de determinada informação de uma organização, Verrecchia (2001) propôs a categorização das pesquisas em disclosure sobre três abordagens distintas relacionadas aos trabalhos analisados. Essas abordagens são conceituadas como: (i) divulgação baseada em associação; (ii) divulgação baseada em julgamento e; (iii) divulgação baseada na eficiência.

Nesse sentido, a primeira abordagem, definida como divulgação baseada em associação, refere-se ao impacto da evidenciação de informações sobre respectivo valor e o volume de negociação de determinado ativo. Assim, a divulgação de informações está associada ao estímulo de se verificar como a divulgação está relacionada com o interesse de mudança no comportamento dos investidores, que buscam no mercado de capitais um meio de maximização de valor (Verrecchia, 2001).

A segunda abordagem é conceituada como divulgação baseada em julgamento, por meio da qual se busca verificar o poder discricionário dos gestores sobre as informações pelo qual detenham conhecimento, isto é, de que maneira se orientam por um comportamento de realizar a divulgação dessas informações (Verrecchia, 2001). 
Nessa perspectiva, considera-se que um investidor racional compreende uma informação retida como sendo uma informação desfavorável sobre o desempenho da organização. Além disso, há um ponto de equilíbrio observado pelos gestores, concernente aos benefícios da divulgação e os respectivos custos associados (Verrecchia, 2001).

Por fim, a terceira abordagem apresentada é definida como "divulgação baseada na eficiência", uma vez que parte do pressuposto de discutir sobre os fatores que influenciam no comportamento em analisar a eficiência das informações a serem evidenciadas. Além disso, considera-se sobre essa abordagem que o comportamento dos gestores sobre realização da divulgação é direcionado pela divulgação antes mesmo de conhecê-la previamente (Verrecchia, 2001).

Destarte, essa pesquisa está delimitada sobre a abordagem baseada na divulgação discricionária, isto é, divulgação de informações voluntárias com base no julgamento dos gestores. Nessa perspectiva, cabe ressaltar que, segundo Verrecchia (2001), o objetivo de um gestor é a maximização de valor da empresa e, com isso, o julgamento entre a divulgação ou não de informações particulares está associado aos fatores determinantes que influenciem positivamente a percepção dos investidores sobre o desempenho e consequentemente o valor da organização.

Sendo assim, emerge uma questão fundamental na Contabilidade, caracterizada pelas escolhas contábeis, isto é, qualquer decisão tomada pelos gestores visando o principal propósito de influenciar o resultado de uma determinada organização (Fields, Lys, \& Vincent, 2001). Cabe ressaltar que, além das escolhas contábeis influenciarem os resultados das organizações, pode dentre outros fatores, induzir o comportamento dos gestores sobre a prática da divulgação de informações (Fields et al., 2001).

Tendo em vista o exposto, evidencia-se a necessidade de compreender a motivação dos gestores sobre a prática das escolhas contábeis, considerando que não são adotadas sem nenhuma finalidade, uma vez que os indivíduos são estimulados por incentivos econômicos (Murcia, Souza, Wuergues, \& Duarte, 2013).

Pontua-se, que os gestores das sociedades por ações, segundo Gitman (2010), em sua maioria não são os proprietários, e, dessa maneira, espera-se que suas tomadas de decisões estejam direcionadas para a maximização desses acionistas. Na medida em que os gestores, por meio de suas tomadas de decisões consigam atingir o interesse dos acionistas, isto é, a maximização do lucro da empresa, por consequência atingirá seus objetivos profissionais.

Isto posto, na próxima seção são discutidos os estudos anteriores utilizados como base para formulação das hipóteses.

\subsection{Estudos empíricos anteriores e desenvolvimento das hipóteses}

A avaliação do desempenho dos gestores, segundo Gitman (2010) pode ser verificada por meio dos indicadores de desempenho econômico e financeiro das organizações. Dessa maneira, esses indicadores poderão contribuir para os usuários verificarem se a organização está sendo dirigida no intuito de atingir seus principais objetivos, isto é, preservar e maximizar o valor dos investidores (Gitman, 2010).

Nesse contexto, observou-se nos estudos anteriores que os indicadores de desempenho econômico e financeiro, modelados e, consequentemente estatisticamente significantes, com influência positiva sobre a divulgação voluntária são relativos à rentabilidade com base nos estudos de Murcia (2009), Alfaraih e Alanezi (2011), Rufino e Monte (2014), Dolinšek e Lutar-Skerbinjek (2018) e Nguyen et al. (2020) e sobre o endividamento, baseado nos resultados encontrados pelos autores Murcia (2009) e Kateb (2012). A partir dessas evidências verificadas nesses estudos, são apresentadas a primeira e a segunda hipótese da pesquisa:

$\mathrm{H}_{1}$ : a rentabilidade (quanto maior) influencia positivamente a probabilidade de as empresas realizarem a prática da divulgação voluntária de informações financeiras. 
$\mathrm{H}_{2}$ : o endividamento (quanto maior) influencia positivamente a probabilidade de as empresas realizarem a prática da divulgação voluntária de informações financeiras.

Além disso, baseado na estrutura organizacional das empresas e, partindo do pressuposto de que aquelas maiores possuem uma sistemática de gestão mais robusta, e, consequentemente, menos custos para dispender na prática da divulgação de informações (Murcia, 2009; Cruz \& Lima, 2010), o tamanho da empresa apresentou influência positiva na divulgação de informações, nos achados de Mendes-da-Silva e Magalhães Filho (2005), Luz et al. (2006), Murcia e Santos (2009b), Cruz e Lima (2010), Kateb (2012), Barac et al. (2014), Thoresson e Niléhn (2014), Rufino e Monte (2014), Hieu e Lan (2015), Forte et al. (2016), Mota e Pinto (2017), Dolinšek e LutarSkerbinjek (2018) e Nguyen et al. (2020).

Baseado nessa evidência tem-se a terceira hipótese da pesquisa.

$\mathrm{H}_{3}$ : o tamanho da empresa (quanto maior) influencia positivamente a probabilidade de as empresas realizarem a prática da divulgação voluntária de informações financeiras.

Além disso, direcionada pela maximização da transparência, considera-se nessa pesquisa, a importância da Governança Corporativa que de acordo com Silveira (2010), desde o final da década de 1990 vem ganhando expressividade no cenário brasileiro. Essa abordagem é motivada pelos recorrentes conflitos entre os agentes das organizações, sobre determinadas práticas particulares de gestão adotadas pelos executivos, consideradas pelos investidores como destruidoras de valor (Silveira, 2010).

Com base nesse comportamento destruidor de valor, acentua-se que as exigências sobre a publicação de determinadas informações evidenciam algumas questões negativas, envolvendo os derivativos no mercado nacional, por exemplo, as companhias Sadia e Aracruz que, na perspectiva de Murcia e Santos (2009a) pelo tratamento indevido aos derivativos obtiveram prejuízos significativos. Assim, esses prejuízos afetaram as operações das respectivas empresas, haja visto que, na Sadia atingiu o maior resultado negativo no período histórico da companhia e a Aracruz teve por parte da empresa responsável pela auditoria a informação sobre a possível descontinuidade (Murcia \& Santos, 2009a).

No intuito de fomentar o desenvolvimento do mercado de capitais brasileiro, a Brasil Bolsa Balcão criou os segmentos especiais de listagem, para classificar as companhias de acordo com os diferentes perfis, definidos como Bovespa Mais, Bovespa Mais Nível 2, Novo Mercado, Nível 2 e Nível 1 (Brasil Bolsa Balcão, 2017). Evidentemente, a exigência pelo maior nível de transparência varia de acordo com os níveis de listagem da Brasil Bolsa Balcão.

Mediante o exposto, sobre a expectativa da governança corporativa na maximização do nível de transparência das organizações, se tem a quarta hipótese da pesquisa.

$\mathrm{H}_{4}$ : o nível de governança corporativa (Novo Mercado) influencia positivamente a probabi-

lidade de as empresas realizarem a prática da divulgação voluntária de informações financeiras.

Por fim, considera-se também nessa pesquisa que umas das principais inquietações no campo do mercado de capitais, consiste na busca pela identificação de fraudes, haja visto eventos ocorridos nesse sentido com grandes organizações e plenamente divulgados por meio das mídias (Assing, Alberton, \& Tesch, 2008). Sobre esse aspecto, emerge o papel das empresas de autoria independente, exercido por meio de seus auditores independentes.

Consoante aos propósitos dos mecanismos de Governança Corporativa, pautados na maximização da transparência e segurança aos investidores, os auditores independentes, de acordo com Zagonov, Pettinicchio e Salganik-Shoshan (2017) possuem a responsabilidade de supervisionar as práticas de gestão adotadas pelos gestores e emitir com base nisso, os seus pareceres aos usuários dessas informações. 
Destarte, a auditoria independente também foi um fator que apresentou influência positiva sobre a prática da divulgação voluntária de informações, encontrada nos estudos de Murcia (2009) e Al-Janadi et al. (2013). Deste modo, formula-se a quinta e última hipótese da pesquisa.

H5: a auditoria (Big Four) influencia positivamente a probabilidade de as empresas realizarem a prática da divulgação voluntária de informações financeiras.

A seguir, na próxima seção, são apresentados os procedimentos metodológicos empregados para o desenvolvimento da pesquisa, com o propósito de atingir o objetivo e responder à questão do estudo.

\section{METODOLOGIA}

Para a definição da amostra, foram coletadas inicialmente informações de todas as companhias não financeiras listadas na Brasil Bolsa Balcão no ano de 2016, totalizando 302. Salienta-se que foram excluídas as companhias do setor financeiro pelas peculiaridades de suas operações (Murcia, 2009). Entretanto, após a análise em profundidade dos relatórios divulgados, para estimação dos modelos, foram desconsideradas da amostra 35 companhias, por apresentar um índice de rentabilidade não condizente com a realidade. Estas empresas apresentaram valor negativo, no período analisado, tanto para o lucro líquido quanto para o patrimônio líquido; resultando assim, em rentabilidade com sinal positivo. Sendo assim, os modelos de regressão propostos na pesquisa foram estimados com 267 observações.

Quanto à coleta dados, para a variável dependente (divulgação ou não de informações voluntária) foi junto aos relatórios financeiros divulgados voluntariamente nos websites das empresas selecionadas na amostra. Por não serem obrigatórios, os relatórios são elaborados de forma estrutural e informacional sob a perspectiva de cada organização e disponibilizados em seus websites como informações complementares. Essas publicações foram consideradas em sua maioria pelas companhias como "press release" ou "earnings release".

Para análise das informações financeiras divulgadas voluntariamente pelas companhias, realizou-se a análise do conteúdo nos relatórios divulgados voluntariamente (Bardin, 2010). A análise de conteúdo sobre o relatório financeiro de cada companhia, referente ao exercício contábil de 2016, ocorreu no período de março a setembro de 2017. A escolha do ano de 2016 se justifica por apresentar o exercício contábil mais recente quando dá realização das análises e, por se acreditar que as empresas, em meio ao turbilhão vivenciado pelo mercado, que desde o ano de 2014, verificou diversas operações de combate a fraudes, dentre elas, a operação Lava-jato, possam ter se preocupado com a questão da transparência em suas informações voluntárias.

Destarte, buscou-se nos relatórios financeiros das empresas, divulgados voluntariamente, cada uma das informações apresentadas na Tabela 1 e, na medida em que foi encontrada a respectiva informação pontuou-se 1, ou 0 caso contrário. Destaca-se, que essa abordagem foi utilizada na pesquisa de Murcia (2009).

Tabela 1 - Categoria e unidades de análise

\begin{tabular}{l|l}
\hline Categoria de análise & \multicolumn{1}{c}{ Unidades de análise (informações detalhadas) } \\
\hline \multirow{5}{*}{ Relatórios Financeiros } & Custos dos produtos/serviços \\
\cline { 2 - 2 } & Preço ou valorização (desvalorização) das ações por tipo \\
\cline { 2 - 2 } & Valor de mercado \\
\cline { 2 - 2 } & Projeções (fluxo de caixa, vendas, lucros) \\
\cline { 2 - 2 } & Indicadores de rentabilidade (ROA/ROE) \\
\cline { 2 - 2 } & Indicadores de liquidez (liquidez seca, liquidez corrente) \\
\cline { 2 - 2 } & Indicadores de endividamento (pl/passivo, pc/pelp) \\
\cline { 2 - 2 } & EBITDA \\
\hline
\end{tabular}

Fonte: Adaptado de Murcia (2009) 
Entretanto, no intuito de avançar as discussões verificadas nos estudos anteriores, na medida em que a empresa evidenciou a informação, realizou-se uma análise em profundidade dessas respectivas informações publicadas sobre a abordagem qualitativa. Isto é, estabeleceu-se uma métrica sobre a profundidade e/ou clareza com que a informação estava disponibilizada.

Cabe ressaltar, que a métrica definida para qualificar as informações, foi construída com o propósito de verificar a transparência entre as informações publicadas pelas companhias. Com isso, visando o nível informacional, as variáveis dependentes dos modelos de regressão logit assumiu 1 para a informação publicada com detalhes, e zero caso contrário. Isso significa que, foram consideradas como informações publicadas com detalhes, aquelas que continham gráficos e/ou ilustrações apresentado séries temporais, ou que continham comentários explicativos.

Com relação às variáveis explicativas, a coleta de dados para o índice de rentabilidade, o índice de endividamento e o tamanho da empresa se deu na base de dados da Economática ${ }^{\circledR}$, e os dados referentes à auditoria e ao nível de governança corporativa, foram coletados na base de dados da Brasil Bolsa Balcão. Destaca-se, que para a construção das variáveis independentes associadas à rentabilidade, ao nível de endividamento e ao tamanho da empresa, foram coletados os valores brutos e não esses índices já calculados, isto é, foram coletados os valores para cálculo dos respetivos indicadores no software estatístico Stata/IC 15.

No tocante a variável independente dicotômica, associada à governança corporativa, foi considerado peso 1 quando a companhia estava na listagem do Novo Mercado e zero caso contrário. Já a variável independente dicotômica, associada à auditoria, foi considerado peso 1 quando a companhia estava auditada pelas "Big Four" e, zero caso contrário.

$\mathrm{Na}$ Tabela 2, são apresentadas às variáveis explicativas, bem como as equações empregadas para os respectivos cálculos e as relações esperadas.

Tabela 2 - Variáveis explicativas

\begin{tabular}{|c|c|c|c|}
\hline Indicador & Procedimentos & Relação Esperada & Fonte \\
\hline Rentabilidade & Rent $=\frac{L L}{P L}$ & Positiva & $\begin{array}{c}\text { Murcia (2009) } \\
\text { Alfaraih e Alanezi (2011) } \\
\text { Rufino e Monte (2014) }\end{array}$ \\
\hline Endividamento & Endiv $=\frac{P C+P \tilde{\mathrm{N}} C}{A T}$ & Positiva & $\begin{array}{c}\text { Murcia (2009) } \\
\text { Kateb (2012) }\end{array}$ \\
\hline Tamanho da Empresa & $A T$ (Total do ativo) & Positiva & $\begin{array}{c}\text { Mendes-da-Silva e Magalhães } \\
\text { Filho (2005) } \\
\text { Luz et al. (2006) } \\
\text { Murcia e Santos (2009b) } \\
\text { Cruz e Lima (2010) } \\
\text { Kateb (2012) } \\
\text { Barac et al. (2014) } \\
\text { Rufino e Monte (2014) } \\
\text { Hieu e Lan (2015) } \\
\text { Forte et al. (2016) } \\
\text { Mota e Pinto (2017) } \\
\text { Thoresson e Niléhn (2014) } \\
\text { Nguyen et al. (2020) }\end{array}$ \\
\hline Governança Corporativa & $\begin{array}{l}\text { Gover (Dummy, } 1 \text { para } \\
\text { empresa listada no novo } \\
\text { mercado, } 0 \text { caso contrário) }\end{array}$ & Positiva & Murcia (2009) \\
\hline Auditoria & $\begin{array}{l}\text { Audit (Dummy, } 1 \text { para } \\
\text { empresa auditada pelas Big } \\
\text { Four, } 0 \text { caso contrário) }\end{array}$ & Positiva & $\begin{array}{c}\text { Murcia (2009) } \\
\text { Al-Janadi et al. (2013) }\end{array}$ \\
\hline
\end{tabular}

Fonte: Dados da pesquisa (2020) 
Para analisar modelos regressivos com variáveis dependentes dicotômicas, Greene (2003) sugere a utilização da modelagem probit ou logit, modelos que permitem realizar estimativas de probabilidade, efeitos marginais, além de uma série de resultados auxiliares. Entretanto, o que diferencia a estimação de cada um dos respectivos modelos, são os pressupostos fundamentais, definido pela distribuição normal ou logística dos dados (Greene, 2003).

Destaca-se ainda, em se tratando da escolha pela utilização de uma variável dummy como variável dependente, um aspecto fundamental da utilização de variável dummy é a oportunidade de qualificar as variáveis, pela ausência do atributo caracterizado na outra categoria. Esse procedimento se realizado pela atribuição de valores aleatórios estaria equivocado, uma vez que os pesos estariam sendo definidos arbitrariamente (Fávero, Belfiore, Silva \& Chan, 2009).

Sendo assim, as análises dos dados coletados foram realizadas por meio de oito modelos de regressão logit, para estimação da probabilidade de as companhias em questão evidenciarem cada uma das informações analisadas nos relatórios divulgados voluntariamente, no intuito de satisfazer a questão norteadora da pesquisa. Isto posto, os modelos propostos na pesquisa são os seguintes.

$$
\begin{aligned}
& \ln \left(\frac{\text { DivCust }_{i}}{1-\text { DivCust }_{i}}\right)=f\left(\beta_{0}+\beta_{1} \text { Renta }_{i}+\beta_{2} \text { Endivid }_{i}+\beta_{3} \text { Tam }_{i}+\beta_{4} \text { Gover }_{i}+\beta_{5} \text { Audit }_{i}+\varepsilon_{i}\right) \\
& \ln \left(\frac{\text { DivPreco }_{i}}{1-\text { DivPreco }_{i}}\right)=f\left(\beta_{0}+\beta_{1} \text { Renta }_{i}+\beta_{2} \text { Endivid }_{i}+\beta_{3} \text { Tam }_{i}+\beta_{4} \text { Gover }_{i}+\beta_{5} \text { Audit }_{i}+\varepsilon_{i}\right) \\
& \ln \left(\frac{\text { DivValor }_{i}}{1-\text { DivValor }_{i}}\right)=f\left(\beta_{0}+\beta_{1} \text { Renta }_{i}+\beta_{2} \text { Endivid }_{i}+\beta_{3} \text { Tam }_{i}+\beta_{4} \text { Gover }_{i}+\beta_{5} \text { Audit }_{i}+\varepsilon_{i}\right) \\
& \ln \left(\frac{\text { DivPro }_{i}}{1-\text { DivPro }_{i}}\right)=f\left(\beta_{0}+\beta_{1} \text { Renta }_{i}+\beta_{2} \text { Endivid }_{i}+\beta_{3} \text { Tam }_{i}+\beta_{4} \text { Gover }_{i}+\beta_{5} \text { Audit }_{i}+\varepsilon_{i}\right) \\
& \ln \left(\frac{\text { DivRent }_{i}}{1-\text { DivRent }_{i}}\right)=f\left(\beta_{0}+\beta_{1} \text { Renta }_{i}+\beta_{2} \text { Endivid }_{i}+\beta_{3} \text { Tam }_{i}+\beta_{4} \text { Gover }_{i}+\beta_{5} \text { Audit }_{i}+\varepsilon_{i}\right) \\
& \ln \left(\frac{\text { DivLiquid }_{i}}{1-\text { DivLiquid }_{i}}\right)=f\left(\beta_{0}+\beta_{1} \text { Renta }_{i}+\beta_{2} \text { Endivid }_{i}+\beta_{3} \text { Tam }_{i}+\beta_{4} \text { Gover }_{i}+\beta_{5} \text { Audit }_{i}+\varepsilon_{i}\right) \\
& \ln \left(\frac{\text { DivEndiv }_{i}}{1-\text { DivEndiv }_{i}}\right)=f\left(\beta_{0}+\beta_{1} \text { Renta }_{i}+\beta_{2} \text { Endivid }_{i}+\beta_{3} \text { Tam }_{i}+\beta_{4} \text { Gover }_{i}+\beta_{5} \text { Audit }_{i}+\varepsilon_{i}\right) \\
& \ln \left(\frac{\text { DivEbitda }_{i}}{1-\text { DivEbitda }_{i}}\right)=f\left(\beta_{0}+\beta_{1} \text { Renta }_{i}+\beta_{2} \text { Endivid }_{i}+\beta_{3} \text { Tam }_{i}+\beta_{4} \text { Gover }_{i}+\beta_{5} \text { Audit }_{i}+\varepsilon_{i}\right)
\end{aligned}
$$

Em que:

DivCust $=$ Divulgação detalhada sobre os custos; DivPreco $=$ Divulgação detalhada sobre o preço das ações; DivValor = Divulgação detalhada sobre o valor de mercado; DivProj = Divulgação detalhada sobre as projeções; DivRent = Divulgação detalhada sobre a rentabilidade; DivLiquid = Divulgação detalhada sobre a liquidez; DivEndiv= Divulgação detalhada sobre o endividamento; DivEbitda = Divulgação detalhada sobre o EBITDA; $f=$ função logística; $\beta_{0}=$ Constante; Renta = Rentabilidade; Endivid = Endividamento; Tam = Tamanho; Gover = Governança; Audit $=$ Auditoria; e $\varepsilon=$ Erro.

A modelagem com variáveis dicotômicas é usualmente estimada pelo método de máxima verossimilhança (Greene, 2003). Considerando que os dados em questão são individuais, coletados para cada empresa em um mesmo instante de tempo, a proposta da presente pesquisa é estimar a probabilidade de uma empresa divulgar uma informação voluntária considerando um conjunto de resultados contábeis (matriz de variáveis explicativas).

\section{ANÁLISE E DISCUSSÃO DOS RESULTADOS}

Nessa seção são apresentados os resultados obtidos por meio da estimação dos modelos propostos. Com isso, a estatística descritiva dos dados relativos às variáveis explicativas contínuas dos modelos de regressão logit estimados é detalhada na Tabela 3, com as 267 observações consideradas na amostra. 
Tabela 3 - Estatística descritiva das variáveis explicativas contínuas

\begin{tabular}{l|c|c|c}
$\begin{array}{c}\text { Variáveis } \\
\text { Explicativas }\end{array}$ & Rentabilidade & Endividamento & Tamanho (mi) \\
\hline Média & $-0,03287$ & 0,76509 & 12,898 \\
\hline Máximo & 13,97243 & 40,79630 & 804,945 \\
\hline Mínimo & $-9,74417$ & 0,04930 & 0,062 \\
\hline Desvio Padrão & 1,27280 & 2,48123 & 55275,69 \\
\hline Observações & 267 & 267 & 267 \\
\hline Jarque-Bera & $5,5 \mathrm{e}+04$ & $7,2 \mathrm{e}+05$ & $2,9 \mathrm{e}+05$ \\
\hline p-valor & 0,0000 & 0,0000 & 0,0000 \\
\hline
\end{tabular}

Fonte: Dados da pesquisa (2020)

Observa-se na Tabela 3 que percentualmente, em média, as empresas possuem rentabilidade negativa (3,29\%). Com relação ao endividamento das 267 companhias analisadas, o percentual médio encontrado foi de aproximadamente $76,51 \%$. Esse percentual encontrado evidencia um alto nível de endividamento médio das companhias brasileiras, listadas na bolsa de valores. Essa discrepância entre os valores encontrados, também foi verificada no tamanho. Sendo assim, verificouse com base no teste de normalidade de Jarque-Bera, que os dados encontrados referentes às variáveis em questão não apresentaram normalidade, na medida em que a hipótese nula do respectivo teste foi rejeitada a 1\% de significância.

Em se tratando das demais variáveis explicativas, definidas como variáveis binárias nos modelos de regressão estimados, a seguir na Tabela 4, são detalhadas cada uma delas.

\begin{tabular}{|c|c|c|}
\hline $\begin{array}{l}\text { Variáveis } \\
\text { Explicativas }\end{array}$ & Governança & Auditoria \\
\hline Valor (1) & 149 & 205 \\
\hline Valor $(0)$ & 118 & 62 \\
\hline Valor (1) & $55,8 \%$ & $76,8 \%$ \\
\hline Valor (0) & $44,2 \%$ & $23,2 \%$ \\
\hline Observações & 267 & 267 \\
\hline
\end{tabular}

Fonte: Dados da pesquisa (2020)

É possível verificar na Tabela 4 que em torno de 55,8\% das 267 companhias analisadas, isto é, aproximadamente metade das companhias não financeiras está listada no segmento de governança corporativa do novo mercado da Brasil Bolsa Balcão. Pontua-se diante dessa evidência encontrada, que ainda há muito a percorrer para que o mercado brasileiro possa avançar nesse quesito. Além disso, verificou-se também, conforme apresentado na Tabela 4, que em torno de 76,8\% dessas companhias são auditadas pelas empresas de auditoria consideradas como "Big Four".

Nessa perspectiva, na Tabela 5 são apresentados os resultados dos coeficientes estimados pelos modelos de regressão logit. Em coluna se tem cada um dos modelos estimados com as cinco (5) variáveis explicativas, apresentadas em linha.

Destaca-se que, segundo Gujarati e Porter (2011), o sinal obtido pelo coeficiente estimado do modelo logit define o acréscimo ou decréscimo na probabilidade. Assim sendo, quando o coeficiente estimado do respectivo modelo de regressão apresenta o sinal positivo, significa um acréscimo na probabilidade ou um decréscimo, caso contrário. 
Tabela 5 - Estimação dos modelos de regressão logit

\begin{tabular}{|c|c|c|c|c|c|c|c|c|}
\hline \multirow{2}{*}{$\begin{array}{l}\text { Variáveis } \\
\text { Explicativas }\end{array}$} & \multicolumn{8}{|c|}{ Variáveis Dependentes (Modelos estimados) } \\
\hline & $\begin{array}{l}\text { DivCust } \\
\text { (I) }\end{array}$ & $\begin{array}{c}\text { DivPreco } \\
\text { (II) }\end{array}$ & $\begin{array}{l}\text { DivValor } \\
\text { (III) }\end{array}$ & $\begin{array}{l}\text { DivProj } \\
\text { (IV) }\end{array}$ & $\begin{array}{l}\text { DivRent } \\
\text { (V) }\end{array}$ & $\begin{array}{l}\text { DivLiquid } \\
\text { (VI) }\end{array}$ & $\begin{array}{l}\text { DivEndiv } \\
\text { (VII) }\end{array}$ & $\begin{array}{l}\text { DivEbitda } \\
\text { (VIII) }\end{array}$ \\
\hline C & $\begin{array}{c}-1,900 \\
(0,00)\end{array}$ & $\begin{array}{l}-2,876 \\
(0,00)\end{array}$ & $\begin{array}{l}-4,906 \\
(0,00)\end{array}$ & $\begin{array}{c}-2,493 \\
(0,00) \\
\end{array}$ & $\begin{array}{l}-3,461 \\
(0,00)\end{array}$ & $\begin{array}{r}-2,890 \\
(0,00) \\
\end{array}$ & $\begin{array}{r}-1,451 \\
(0,00) \\
\end{array}$ & $\begin{array}{r}-1,700 \\
(0,00) \\
\end{array}$ \\
\hline RENTA & $\begin{array}{l}-0,206 \\
(0,86)\end{array}$ & $\begin{array}{l}0,212 \\
(0,11)\end{array}$ & $\begin{array}{l}0,083 \\
(0,73)\end{array}$ & $\begin{array}{l}0,037 \\
(0,77)\end{array}$ & $\begin{array}{l}0,052 \\
(0,74)\end{array}$ & $\begin{array}{l}0,049 \\
(0,76)\end{array}$ & $\begin{array}{l}-0,061 \\
(0,59)\end{array}$ & $\begin{array}{l}-0,197 \\
(0,17)\end{array}$ \\
\hline ENDIVID & $\begin{array}{l}-0,482 \\
(0,68)\end{array}$ & $\begin{array}{l}-0,054 \\
(0,74)\end{array}$ & $\begin{array}{l}-0,197 \\
(0,84)\end{array}$ & $\begin{array}{l}-0,022 \\
(0,82)\end{array}$ & $\begin{array}{l}-0,504 \\
(0,50)\end{array}$ & $\begin{array}{l}-0,014 \\
(0,91)\end{array}$ & $\begin{array}{l}-0,054 \\
(0,56)\end{array}$ & $\begin{array}{l}-0,063 \\
(0,59)\end{array}$ \\
\hline TAM & $\begin{array}{l}6 \mathrm{E}-04 \\
(0,00)^{*}\end{array}$ & $\begin{array}{c}-1 \mathrm{E}-06 \\
(0,76)\end{array}$ & $\begin{array}{l}4 \mathrm{E}-06 \\
(0,19)\end{array}$ & $\begin{array}{l}\mathrm{E}-07 \\
(0,87)\end{array}$ & $\begin{array}{l}-3 \mathrm{E}-07 \\
(0,94)\end{array}$ & $\begin{array}{l}4 \mathrm{E}-07 \\
(0,92)\end{array}$ & $\begin{array}{l}5 \mathrm{E}-07 \\
(0,84)\end{array}$ & $\begin{array}{c}\text { 7E-04 } \\
(0,01)^{* * *}\end{array}$ \\
\hline GOVER & $\begin{array}{c}1,256 \\
(0,00)^{*}\end{array}$ & $\begin{array}{c}2,442 \\
(0,00)^{*}\end{array}$ & $\begin{array}{c}1,966 \\
(0,01)^{* *}\end{array}$ & $\begin{array}{c}0,869 \\
(0,01)^{* *}\end{array}$ & $\begin{array}{c}1,133 \\
(0,00)^{*}\end{array}$ & $\begin{array}{c}1,298 \\
(0,00)^{*}\end{array}$ & $\begin{array}{c}1,550 \\
(0,00)^{*}\end{array}$ & $\begin{array}{c}2,414 \\
(0,00)^{*}\end{array}$ \\
\hline AUDIT & $\begin{array}{c}0,765 \\
(0,04)^{* *}\end{array}$ & $\begin{array}{c}1,210 \\
(0,00)^{*}\end{array}$ & $\begin{array}{l}1,101 \\
(0,30) \\
\end{array}$ & $\begin{array}{c}0,840 \\
(0,08)^{* * * *}\end{array}$ & $\begin{array}{c}1,518 \\
(0,04)^{* *}\end{array}$ & $\begin{array}{l}0,202 \\
(0,71) \\
\end{array}$ & $\begin{array}{c}1,139 \\
(0,00)^{*}\end{array}$ & $\begin{array}{c}1,200 \\
(0,00)^{*}\end{array}$ \\
\hline $\begin{array}{c}\mathrm{R}^{2} \text { McFad- } \\
\text { den }\end{array}$ & 0,20 & 0,26 & 0,11 & 0,05 & 0,09 & 0,05 & 0,17 & 0,36 \\
\hline LR Stat. & $\begin{array}{l}71,96 \\
(0,00) \\
\end{array}$ & $\begin{array}{l}93,20 \\
(0,00) \\
\end{array}$ & $\begin{array}{l}15,19 \\
(0,00)\end{array}$ & $\begin{array}{l}15,22 \\
(0,00) \\
\end{array}$ & $\begin{array}{l}21,16 \\
(0,00) \\
\end{array}$ & $\begin{array}{l}11,49 \\
(0,04) \\
\end{array}$ & $\begin{array}{l}60,63 \\
(0,00) \\
\end{array}$ & $\begin{array}{l}124,4 \\
(0,00) \\
\end{array}$ \\
\hline $\begin{array}{c}\text { Obs. (0) } \\
\%\end{array}$ & $\begin{array}{l}144 \\
54 \% \\
\end{array}$ & $\begin{array}{c}158 \\
59 \% \\
\end{array}$ & $\begin{array}{l}247 \\
93 \% \\
\end{array}$ & $\begin{array}{c}208 \\
78 \% \\
\end{array}$ & $\begin{array}{l}225 \\
86 \% \\
\end{array}$ & $\begin{array}{c}231 \\
86 \% \\
\end{array}$ & $\begin{array}{c}118 \\
44 \% \\
\end{array}$ & $\begin{array}{c}93 \\
35 \% \\
\end{array}$ \\
\hline $\begin{array}{c}\text { Obs. (1) } \\
\%\end{array}$ & $\begin{array}{r}123 \\
46 \% \\
\end{array}$ & $\begin{array}{r}109 \\
41 \% \\
\end{array}$ & $\begin{array}{l}20 \\
7 \% \\
\end{array}$ & $\begin{array}{c}59 \\
22 \% \\
\end{array}$ & $\begin{array}{c}42 \\
14 \% \\
\end{array}$ & $\begin{array}{c}36 \\
14 \% \\
\end{array}$ & $\begin{array}{r}149 \\
56 \% \\
\end{array}$ & $\begin{array}{l}174 \\
65 \% \\
\end{array}$ \\
\hline Total Obs. & 267 & 267 & 267 & 267 & 267 & 267 & 267 & 267 \\
\hline
\end{tabular}

Variáveis:

DivCust = Divulgação detalhada sobre os custos; DivPreco = Divulgação detalhada sobre o preço das ações; DivValor = Divulgação detalhada sobre o valor de mercado; $\boldsymbol{D i v P r o j}=$ Divulgação detalhada sobre as projeções; $\boldsymbol{D i v R e n t}=$ Divulgação detalhada sobre a rentabilidade; DivLiquid $=$ Divulgação detalhada sobre a li-

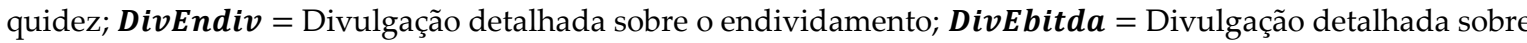
o EBITDA; C = Constante; Renta = Rentabilidade; Endivid $=$ Endividamento Tam = Tamanho; Gover $=$ Governança; e Audit = Auditoria.

* significância estatística ao nível de 1\%

** significância estatística ao nível de 5\%

*** significância estatística ao nível de 10\%

Fonte: Dados da pesquisa (2020)

Verifica-se, com base nos resultados apresentados na Tabela 5, que todos os modelos estimados passaram pelo teste da razão de verossimilhança (RV). Nesse teste, a hipótese nula é de que os coeficientes angulares do modelo de regressão são simultaneamente iguais a zero (Gujarati \& Porter, 2011). Acentua-se apenas que no modelo de regressão referente à divulgação de informações sobre a liquidez das empresas a significância estatística foi ao nível de 5\%. Já nos demais modelos estimados, a significância estatística foi ao nível de $1 \%$.

Ao final da Tabela 5 foram apresentadas as ocorrências das variáveis dicotômicas 1 e 0 para cada modelo estimado. Essa informação é relevante, pois contribui para entender a representatividade da divulgação para cada variável explicativa. Observa-se que a variável dependente DivEbidta é a que tem o maior número de empresas a divulgando informações (65\%), seguida pela variável endividamento (56\%).

As variáveis "divulgação detalhada sobre custo" e "preço das ações", também foram representativas, respectivamente 46 e $41 \%$. Para as demais variáveis explicativas (projeções, rentabilidade, liquidez e valor de mercado) a ocorrência do 1, divulgar detalhadamente tal informação, foi para um número bem menor de empresas. 
Cabe destacar, a importância da variável governança corporativa sobre a decisão de divulgar ou não informações voluntárias. Observa-se que, estatisticamente, o peso dessa informação é expressivamente relevante, pois mesmo com um número significativo de empresas que não divulgam informações, o coeficiente da variável governança corporativa é significativo em todos os modelos.

Nessa perspectiva, verificou-se que a prática de divulgação de informações financeiras voluntárias pelas companhias ainda é significativamente baixa. Cabe ressaltar que, essas observações se referem à divulgação de informações que apresentaram um melhor nível informacional de acordo com o critério estabelecido nesse estudo. Dessa maneira, na Tabela 6 são apresentados os resultados referentes ao teste de validade preditiva dos modelos estimados (teste de HosmerLemeshow).

O teste de Hosmer-Lemeshow é utilizado para analisar o ajuste do modelo de regressão com distribuição logística. Desse modo, o teste realiza a comparação entre as probabilidades ajustadas e as probabilidades observadas, por meio da divisão das observações em dez grupos. Destaca-se que a hipótese nula do teste de Hosmer-Lemeshow é de que não há diferenças entre o ponto previsto e o observado. Para não rejeição da hipótese nula ao nível de $5 \%$ de significância, o $p$ valor deve ser maior que 5\% (Fávero et al., 2009).

Tabela 6 - Validade preditiva dos modelos estimados (Hosmer-Lemeshow)

\begin{tabular}{c|c|c}
\hline Modelo & H-L Statistic & p Valor \\
\hline DivCust (I) & 2,06 & 0,9790 \\
\hline DivPreco (II) & 12,45 & 0,1321 \\
\hline DivValor (III) & 6,26 & 0,6181 \\
\hline DivProj (IV) & 7,61 & 0,4720 \\
\hline DivRent (V) & 9,68 & 0,2882 \\
\hline DivLiquid (VI) & 9,67 & 0,2888 \\
\hline DivEndiv (VII) & 3,04 & 0,9317 \\
\hline DivEbitda (VIII) & 5,74 & 0,6764 \\
\hline
\end{tabular}

Variáveis:

DivCust = Divulgação detalhada sobre os custos; DivPreco = Divulgação detalhada sobre o preço das ações;

DivValor = Divulgação detalhada sobre o valor de mercado; DivProj = Divulgação detalhada sobre as projeções; DivRent $=$ Divulgação detalhada sobre a rentabilidade; DivLiquid $=$ Divulgação detalhada sobre a liquidez; DivEndiv = Divulgação detalhada sobre o endividamento; e DivEbitda = Divulgação detalhada sobre o EBITDA.

Fonte: Dados da pesquisa (2020)

Diante disso, verificou-se por meio da estatística do teste de Hosmer-Lemeshow apresentada na Tabela 6, que em todos os modelos de regressão estimados não se rejeitou a hipótese nula a qualquer nível de significância estatística. Isto é, de acordo com os resultados apresentados por meio do teste de Hosmer-Lemeshow não houve diferença estatística entre o ponto previsto e o observado.

Nesse sentido, observando os resultados apresentados pelos coeficientes associados às variáveis explicativas na Tabela 5, é possível verificar que, em nenhum dos modelos estimados as variáveis associadas à rentabilidade e endividamento se mostraram relevantes para explicar a probabilidade das empresas divulgarem detalhadamente informações voluntárias. Esses achados contrapõe a expectativa e as evidências verificadas por Mendes-da-Silva e Magalhães Filho (2005), Luz et al. (2006), Murcia e Santos (2009b), Cruz e Lima (2010), Kateb (2012), Barac et al. (2014), Thoresson e Niléhn (2014), Rufino e Monte (2014), Hieu e Lan (2015), Forte et al. (2016), Mota e Pinto (2017), Dolinšek e Lutar-Skerbinjek (2018) e Nguyen et al. (2020). 
Diante desses achados, foram rejeitadas as hipóteses $\mathrm{H}_{1}$ e $\mathrm{H}_{2}$ propostas na pesquisa, evidenciando-se que tanto uma melhor rentabilidade quanto um maior endividamento, não alteram a probabilidade de as companhias realizarem a divulgação voluntária de informações financeiras.

O coeficiente associado a variável tamanho da empresa apresentou significância estatística, com influência positiva sobre a probabilidade da divulgação de informações voluntárias, conforme Tabela 5, em dois modelos estimados. Esses achados foram verificados nos modelos DivCust (significância estatística ao nível de 1\%) e DivEbitda (significância estatística ao nível de 5\%), corroborando com a expectativa e as evidências encontradas por Mendes-da-Silva e Magalhães Filho (2005), Luz et al. (2006), Murcia e Santos (2009b), Cruz e Lima (2010), Kateb (2012), Barac et al. (2014), Thoresson e Niléhn (2014), Rufino e Monte (2014), Hieu e Lan (2015), Forte et al. (2016), Mota e Pinto (2017), Dolinšek e Lutar-Skerbinjek (2018) e Nguyen et al. (2020).

Além disso, verificou-se com base nos resultados apresentados pelos coeficientes associados à variável governança corporativa e apresentados na Tabela 5, que em todos os modelos estimados, foram encontradas significância estatística, com influência positiva sobre a probabilidade da divulgação de informações voluntárias. Cabe ressaltar, a significativa influência verificada da governança corporativa, por exemplo, no modelo DivCust, apresentou, aproximadamente $49 \%$ a mais nas chances de divulgação da informação (resultados detalhados e discutidos na Tabela 7).

Com relação aos resultados verificados nos coeficientes associados a variável auditoria e evidenciados na Tabela 5, constatou-se estatisticamente significantes, com influência positiva sobre a probabilidade da divulgação de informações voluntárias em seis modelos estimados. Esses achados foram verificados nos modelos DivPreco, DivEndiv e DivEbitda, com significância estatística ao nível de $1 \%$, respectivamente, e nos modelos DivCust e DivRent, com significância estatística ao nível de 5\%, respectivamente, e no modelo DivProj, com significância estatística ao nível de 10\%, corroborando com a expectativa e as evidências encontradas por Murcia (2009) e Al-Janadi et al. (2013).

Importante relembrar que, diferentemente do Modelo de Probabilidade Linear (MPL), os coeficientes angulares estimados e apresentados na Tabela 5, não fornecem a taxa de variação da probabilidade para uma unidade de variação no regressor. A taxa de variação da probabilidade ou o efeito marginal foi analisado apenas para os coeficientes que se mostraram estatisticamente significativo. Sendo assim, na Tabela 7, são apresentadas as probabilidades oriundas dos coeficientes estimados nos modelos de regressão evidenciados na Tabela 5.

Tabela 7 - Efeito marginal após o modelo logit (taxa de variação da probabilidade)

\begin{tabular}{l|r|r|r|r|r}
\hline \multirow{2}{*}{ MODELO } & \multicolumn{6}{|c}{ VARIÁVEIS EXPLICATIVAS } \\
\cline { 2 - 6 } & \multicolumn{1}{|c|}{ RENTA } & ENDIVID & TAM & \multicolumn{1}{c}{ GOVER } & \multicolumn{1}{c}{ AUDIT } \\
\hline (I) DivCust & $-0,01$ & $-0,01$ & $0,00^{* *}$ & $0,30^{*}$ & $0,19^{* *}$ \\
\hline (II) DivPreco & 0,05 & $-0,01$ & $-0,00$ & $0,49^{*}$ & $0,24^{* *}$ \\
\hline (III) DivValor & 0,00 & $-0,01$ & 0,00 & $0,08^{* *}$ & 0,37 \\
\hline (IV) DivProj & 0,01 & $-0,01$ & 0,00 & $0,14^{*}$ & $0,12^{* *}$ \\
\hline (V) DivRent & 0,01 & $-0,05$ & $-0,00$ & $0,11^{*}$ & $0,12^{*}$ \\
\hline (VI) DivLiquid & 0,01 & $-0,00$ & 0,00 & $0,13^{* *}$ & 0,02 \\
\hline (VII) DivEndiv & $-0,02$ & $-0,01$ & 0,00 & $0,37^{*}$ & $0,28^{*}$ \\
\hline (VIII) DivEbitda & $-0,03$ & $-0,01$ & $0,00^{*}$ & $0,41^{*}$ & $0,23^{* *}$ \\
\hline
\end{tabular}

Variáveis:

DivCust = Divulgação detalhada sobre os custos; DivPreco = Divulgação detalhada sobre o preço das ações; DivValor = Divulgação detalhada sobre o valor de mercado; DivProj = Divulgação detalhada sobre as projeções; DivRent $=$ Divulgação detalhada sobre a rentabilidade; DivLiquid = Divulgação detalhada sobre a liquidez; DivEndiv = Divulgação detalhada sobre o endividamento; DivEbitda = Divulgação detalhada sobre o EBITDA; Renta = Rentabilidade; Endivid = Endividamento; Tam = Tamanho; Gover = Governança; Audit $=$ Auditoria.

* significância estatística ao nível de 1\% 
** significância estatística ao nível de 5\%

*** significância estatística ao nível de 10\%

Fonte: Dados da pesquisa (2020)

Verifica-se, com base nos resultados apresentados na Tabela 7, que o coeficiente associado à variável explicativa governança corporativa, apresentou significância estatística em todos os modelos estimados. Isto é, o nível de governança corporativa influencia positivamente a probabilidade de as empresas realizarem a prática da divulgação voluntária de informações financeiras $\left(\mathrm{H}_{4}\right)$. Isso significa que, a condição das companhias estarem listadas no nível de governança corporativa do Novo Mercado, influenciou positivamente a probabilidade da prática de divulgação voluntária de informações.

Esse resultado é muito importante, pois a influência estatística encontrada entre os coeficientes estimados pelos modelos revelou que a governança corporativa possui uma significativa relevância na probabilidade de os gestores das companhias praticarem a divulgação voluntária. Compreendendo o resultado, é possível afirmar que empresas listadas no nível de governança corporativa do Novo Mercado têm 49\% mais chance de divulgarem informações detalhadas sobre o preço de suas ações. Essas mesmas empresas têm cerca de $40 \%$ a mais de chance de divulgarem detalhadamente informações sobre o EBITDA.

Em ordem de relevância, pela magnitude do efeito marginal, as empresas pertencentes ao Novo Mercado têm 36,7\% a mais de chance de divulgarem informações detalhadas sobre seus custos do que as empresas listadas em outros níveis de governança. O mesmo resultado é para a variável endividamento, com chance $36,7 \%$ superior às empresas não pertencentes ao Novo Mercado.

As empresas pertencentes ao Novo Mercado têm 8,44\% a mais de chance de divulgarem informações detalhadas sobre o seu valor de mercado do que as não pertencentes a esse nível de governança corporativa. Além disso, as empresas pertencentes ao Novo Mercado têm uma chance de $11,08 \%$ a mais de divulgarem informação detalhada sobre a rentabilidade.

No que se refere à liquidez, as empresas listadas no Novo Mercado têm 13\% mais chance do que as não pertencentes a esse nível de governança de divulgarem detalhadamente esta informação. Sobre a variável projeções, as empresas do Novo Mercado têm 13,6\% mais chance de divulgarem informações detalhadas sobre esta variável, do que as não pertencentes ao Novo Mercado.

Mesmo existindo um longo caminho a percorrer para o ingresso de mais empresas no segmento de listagem do Novo Mercado (Tabela 4), os resultados verificados nessa pesquisa, mostram que existe uma distinção na prática de divulgação de informações dessas empresas. Pode-se inferir que a exigência para se pertencer a esse nível de governança corporativa conduz a mais transparência no tocante às informações divulgadas voluntariamente.

Esses resultados encontrados não se contrapõem com os achados de Murcia e Santos (2009b) e Mota e Pinto (2017), na medida em que os achados desses autores não apresentaram significância estatística. Cabendo descartar, no entanto, que os respectivos pesquisadores não estimaram modelos probabilísticos e sim dados em painel com a variável dependente sendo quantitativa.

Por fim, constatou-se que os coeficientes associados à variável auditoria apenas não foram estatisticamente significantes nos modelos referentes à divulgação de informações das empresas sobre o valor de mercado e a liquidez. Sendo assim, com exceção dos respectivos modelos estimados, cujos coeficientes associados à auditoria não foram estatisticamente significantes, com base nos resultados encontrados nos demais modelos estimados, não se rejeitou a hipótese $\mathrm{H}_{5}$.

É importante observar, que ser auditado por uma "Big Four" aumenta em 27,7\% a chance da empresa praticar uma divulgação detalhada sobre o endividamento, comparativamente às empresas não auditadas por "Big Four". A chance das companhias auditadas pelas "Big Four" de di- 
vulgarem detalhadamente o preço das suas ações é 23,8\% maior do que as não auditadas. A menor chance de divulgação encontrada para as respectivas empresas auditadas pelas "Big Four" foi em torno de $12 \%$, no modelo de divulgação de informações sobre a rentabilidade das companhias.

O resultado se repete nos modelos de regressão sobre a publicação das projeções, de maneira que a companhia ser auditada por "Big Four" também maximiza a chance de publicação em aproximadamente $12 \%$ em relação às demais empresas. Esses resultados encontrados corroboram com os achados de Murcia e Santos (2009b) e Al-Janadi et al. (2013).

Contudo, de acordo com os resultados apresentados e discutidos nessa seção, considera-se que dentre os coeficientes estimados e associados às variáveis explicativas propostas na pesquisa por meio dos modelos de regressão logit, distinguiu-se dois dos cinco fatores. Em primeiro lugar, a governança corporativa teve resultado uniforme entre os respectivos modelos de regressão, com influência positiva sobre a probabilidade e, por conseguinte, a auditoria com forte indicativo de influência sobre a probabilidade do disclosure voluntário.

Esses fatores, quando considerados em conjunto nos modelos estimados, por exemplo, no modelo sobre a divulgação de informações detalhadas sobre o preço das ações, incrementam em aproximadamente $73 \%$ as chances das empresas em questão realizarem a prática da divulgação voluntária. As chances conjuntas também são significativas, no modelo de divulgação sobre o EBITDA, em torno de $64 \%$ de incremento sobre a prática da divulgação voluntária.

Mesmo nos modelos referentes a rentabilidade e as projeções das companhias, na qual os coeficientes apresentaram as menores chances para o fator auditoria independente, quando somado com a governança, representam uma significativa influência na prática da divulgação voluntária. Os coeficientes do primeiro modelo evidenciaram incremento na chance de aproximadamente $23 \%$, e consequentemente, os coeficientes do segundo modelo apresentaram incremento na chance de em torno de $26 \%$ das empresas em questão realizarem a divulgação das informações voluntárias analisadas.

Apesar disso, constatou-se ainda, baseado nos achados da pesquisa, que a prática da divulgação de informações voluntárias das companhias em questão, não foi influenciada por fatores relativos a resultados financeiros. Essa evidência verificada levou em consideração a literatura que fundamentou a pesquisa, uma vez que foram modeladas as variáveis explicativas mais utilizadas em estudos anteriores, além dos resultados encontrados por meio dos coeficientes dos modelos de regressão estimados.

Evidentemente que poderia se esperar uma influência positiva da governança corporativa sobre a probabilidade de evidenciação voluntária de informações, fundamentado nas exigências relativas ao segmento do Novo Mercado. Acentua essa constatação, a forte influência obtida nos coeficientes associados à governança corporativa em todos os modelos estimados. A referida constatação confirma que a governança corporativa realmente está cumprindo o papel de promover a transparência nas organizações.

Semelhantemente ao achado sobre a governança corporativa, cabe a ênfase sobre a auditoria independente, que também poderia se esperar a influência positiva. Todavia, mesmo não apresentando significância estatística em todos os modelos estimados, em sua maioria, os coeficientes associados à auditoria independente foram estatisticamente significantes, com uma evidente influência sobre a probabilidade de divulgação comparativamente às empresas não auditadas por uma "Big Four". Assim, pode-se inferir que as "Big Four" podem efetivamente estar promovendo um maior nível de transparência nas organizações.

Entretanto, faz-se necessário ressaltar diante desses resultados, que a modelagem econométrica utilizada nessa pesquisa é distinta daquelas empregadas nos estudos anteriores, principalmente no que tange à natureza dos modelos. Isto é, enquanto na maioria dos estudos anteriores, os 
resultados foram analisados por meio de modelagens do tipo dados em painel, a análise dos dados nesse trabalho foi realizada com a modelagem logit.

Além da distinção entre a natureza da modelagem utilizada nesse estudo, quando comparado com os estudos anteriores, cabe acentuar a particularidade das variáveis dependentes construídas para os modelos analisados. A peculiaridade dessas variáveis deu-se pela construção de uma métrica específica, com o propósito de mensurar a qualidade do conteúdo informacional publicado pelas companhias aos seus usuários.

Por fim, após a discussão dos resultados realizada, na próxima seção são apresentadas as considerações finais sobre a pesquisa, evidenciando os principais aspectos encontrados, bem como direcionamentos para estudos futuros acerca da temática do disclosure voluntário de informações financeiras das empresas.

\section{CONSIDERAÇÕES FINAIS}

O objetivo da pesquisa foi analisar os fatores determinantes da probabilidade da divulgação voluntária de informações financeiras nas companhias brasileiras não financeiras de capital aberto, listadas na Brasil Bolsa Balcão no ano de 2016. Assim, a análise dos dados deu-se por meio de modelos de regressão logit, considerando a qualidade das informações na construção da variável dependente dos respectivos modelos.

Acentua-se que a utilização da modelagem probabilística do tipo logit para análise dos resultados, deu-se pela estrutura da pesquisa, dos dados e do objetivo, isto é, por ser considerada como melhor opção diante da análise proposta. Essa proposição foi confirmada quando analisados os resultados apresentados nos testes de Hosmer-Lemeshow, relativos ao ajuste dos modelos de regressão estimados. Destaca-se que em todos os modelos, a hipótese nula do teste não foi rejeitada a qualquer nível de significância, evidenciando que não há diferenças entre os pontos previstos e os observados, estimados pelos modelos propostos na pesquisa e, que, portanto, os modelos se mostram significantes e relevantes.

Pode-se inferir, diante dos achados, que estar listada no nível de governança corporativa do Novo Mercado é um fator relevante na influência sobre a probabilidade de as companhias realizarem a prática da divulgação voluntária de informações. Com isso, evidenciou-se que a probabilidade de uma organização que está listada no nível de governança corporativa do novo mercado, realizar a prática de divulgação voluntária de informações financeiras é maior, quando comparada aos demais segmentos de listagem de níveis de governança corporativa da Brasil Bolsa Balcão.

Cabe destacar, que uma relevante contribuição da pesquisa está no resultado do coeficiente associado à variável governança corporativa que apresentou significância estatística em todos os modelos estimados, não rejeitando na integralidade a $\mathrm{H}_{4}$. Fundamentado nessa evidência, observase a importância desse fator na questão da divulgação voluntária. Ou seja, mesmo no modelo de regressão sobre divulgação de informações detalhadas sobre o valor de mercado, cuja variável dependente continha apenas $7 \%$ das observações com peso um, o coeficiente foi estatisticamente significante, com uma influência positiva de $8,44 \%$ sobre as chances de publicação.

Outro fator importante verificado nos achados da pesquisa é aquele associado à variável auditoria independente, na medida em que os coeficientes apresentaram significância estatística em seis dos oito modelos estimados. Sendo assim, a empresa ser auditada pelas "Big Four" pode ser uma condição de maior probabilidade de os gestores praticarem o disclosure voluntário de informações. Além disso, a menor influência encontrada nos respectivos coeficientes, em relação à prática de divulgação voluntária foi o incremento de aproximadamente $12 \%$ de chances, no modelo de informações detalhadas sobre a rentabilidade.

Nesse sentido, convém enfatizar que a maximização dos procedimentos visando à transparência das empresas, pode promover, principalmente, a redução da assimetria informacional entre 
os participantes do mercado e, consequentemente, a maximização dos níveis de confiança para investimento nas organizações em questão. Diante do exposto, se defende que essas informações publicadas com detalhes, até então considerada como complementares por não serem obrigatórias, poderiam esclarecer melhor a situação das empresas aos interessados.

No entanto, ressalte-se que se deve ter cuidado em comparar modelos estatísticos que se propõem a análises distintas. Portanto, os resultados encontrados por meio das estimativas realizadas com os modelos de regressão logit propostos na pesquisa, não podem ser generalizados, tornando-se aceitáveis somente para as empresas contidas na amostra.

Evidentemente, como uma característica dos estudos científicos, a pesquisa possui limitações. Uma das limitações verificadas é concernente à amostra da pesquisa, isto é, composto somente pelas organizações não financeiras listadas na Brasil Bolsa Balcão, na medida em que as empresas que não são caracterizadas como uma sociedade anônima não possui obrigatoriedade de publicarem suas informações financeiras. Outras limitações verificadas consistem no efeito apenas em cross-section, no emprego apenas do modelo de regressão logit para os achados e a construção da métrica do disclosure, considerando os aspectos qualitativos das informações publicadas.

Diante disso, no tocante às recomendações para estudos futuros pressupõem-se que o desenvolvimento de pesquisas equivalentes, considerando outros exercícios contábeis, poderia proporcionar a comparabilidade sobre o progresso da probabilidade das empresas sobre a divulgação de informações voluntárias. Além disso, como a métrica utilizada para a construção das variáveis dependentes dos modelos estimados abarcam aspectos de qualidade, poderia ser analisado o comportamento no que se refere ao detalhamento das informações.

\section{REFERÊNCIAS}

Adam, M., Mukhtaruddin, M., \& Putri, R. A. (2014). Earning management, level of voluntary disclosure and cost of equity capital: empirical study on lq-45 index manufacturing companies listed in Indonesia. GSTF Journal on Business Review, 3(3), 8-16.

Alfaraih, M. M., \& Alanezi, F. S. (2011). Does voluntary disclosure level affect the value relevance of accounting information? Accounting \& Taxation, 3(2), 65-84.

Al-Janadi, Y., Rahman, R. A., \& Omar, N. H. (2013). Corporate governance mechanisms and voluntary disclosure in Saudi Arabia. Research Journal of Finance and Accounting, 4(4), 25-35.

Alves, H. S., Canadas, N., \& Rodrigues, A. M. (2015). Voluntary disclosure, information asymmetry and the perception of governance quality: an analysis using a structural equation model. Tékhne, 13(1), 66-79.

Asay, H. S., Elliott, W. B., \& Rennekamp, K. (2016). Disclosure Readability and the Sensitivity of Investors' Valuation Judgments to Outside Information. The Accounting Review, 92(4), 1-25.

Assing, I., Alberton, L., \& Tesch, J. M. (2008). O comportamento das fraudes nas empresas brasileiras. Revista da FAE, 11(2), 141-152.

Barac, Z. A., Granic, M., \& Vuko, T. (2014). The determinants of voluntary disclosure in Croatia. World Academy of Science, Engineering and Technology, International Journal of Social, Behavioral, Educational, Economic, Business and Industrial Engineering, 8(4), 1057-1063.

Bardin, L. (2010). Análise de conteúdo. Lisboa: Edições 70.

Birjandi, H. (2015). The study effect agency theory and signaling theory on the level of voluntary disclosure of listed companies in Tehran Stock Exchange. Research Journal of Finance and Accounting, 6(1), 174-183. 
Brasil Bolsa Balcão. Segmentos de listagem. Recuperado em 27 outubro, 2017 de http://www.bmfbovespa.com.br/pt_br/listagem/acoes/segmentos-de-listagem/sobresegmentos-de-listagem/

Cruz, C. V. O. A., \& Lima, G. A. S. F. (2010). Reputação corporativa e nível de disclosure das empresas de capital aberto no Brasil. Revista Universo Contábil, 6(1), 85-101.

Diamond, D. W., \& Verrecchia, R. E. (1991). Disclosure, liquidity, and the cost of capital. The Journal of Finance, 46(4), 1325-1359.

Dolinšek, T, \& Lutar-Skerbinjek, A. (2018). Voluntary disclosure of financial information on the internet by large companies in Slovenia. Kybernetes, 47(3), 458-473.

Dye, R. A. (1985). Disclosure of nonproprietary information. Journal of Accounting Research, 23(1), 123-145.

Fávero, L. P., Belfiore, P., Silva, F. D., \& Chan, B. L. (2009). Análise de dados: modelagem multivariada para tomada de decisões. Rio de Janeito: Elsevier.

Fields, T. D., Lys, T. Z., \& Vincent, L. (2001). Empirical research on accounting choice. Journal of Accounting and Economics, 31(1), 255-307.

Forte, S. H. A. C., Perez, M. A., Bessa, F. R., \& Oliveira, O. V. (2015). Práticas de disclosure voluntário de informações estratégicas e de índices financeiros de empresas do nordeste brasileiro: uma análise a partir do modelo de Murcia. RACE-Revista de Administração, Contabilidade e Economia, 14(2), 597-622.

Forte, L. M., Santos Neto, J. B., Nobre, F. C., Nobre, L. H. N., \& Queiroz, D. B. (2016). Determinants of voluntary disclosure: a study in the brazilian banking sector. Revista de Gestão, Finanças e Contabilidade, 5(2), 23-37.

Gama Boaventura, J. M., Cardoso, F. R., Silva, E. S., \& Silva, R. S. (2009). Teoria dos stakeholders e teoria da firma: um estudo sobre a hierarquização das funções-objetivo em empresas brasileiras. Revista Brasileira de Gestão de Negócios, 11(32), 289-307.

Gitman, J. L. (2010). Princípios da administração financeira. (12a ed.). Porto Alegre: Bookman.

Greene, W. H. (2003). Econometric analysis. Delhi: Pearson Education India.

Gujarati, D. N., \& Porter, D. C. (2011). Econometria Básica (5a ed.). Porto Alegre: AMGH Editora.

Hieu, P. D., \& Lan, D. T. H. (2015). Factors influencing the voluntary disclosure of Vietnamese listed companies. Journal of Modern Accounting and Auditing, 11(12), 656-676.

Iudícibus, S., Martins, E., \& Carvalho, N. (2005). Contabilidade: aspectos relevantes da epopéia de sua evolução. Revista Contabilidade E Finanças - USP, 16(38), 7-19.

Kateb, I. (2012). An analysis of the determinants of voluntary structural capital disclosure by listed French companies. International Journal of Business and Management, 7(11), 95-110.

Luz, J. J. M., Camargos, M. A., \& Lima, J. O. (2006). Análise da relação entre disseminação voluntária de informações em sites corporativos e desempenho financeiro de empresas brasileiras. REGE Revista de Gestão, 13(4), 11-24.

Madden, B. J. (2017). The Purpose of the Firm, Valuation, and the Management of Intangibles. Journal of Applied Corporate Finance, 29(2), 76-86.

Mendes-da-Silva, W., \& Magalhães Filho, P. A. O. (2005). Determinantes da disseminação voluntária de informações financeiras na internet. $R A E$-eletrônica, 4(2). 
Mota, S. L., \& Pinto, S. K. M. (2017). A utilização do twitter na análise do disclosure voluntário das empresas brasileiras com níveis diferenciados de governança corporativa. Revista Evidenciação Contábil \& Finanças, 5(1), 22-38.

Murcia, F. D. (2009). Fatores determinantes do nível de disclosure voluntário de companhias abertas no Brasil. Tese de Doutorado, Faculdade de Economia, Administração e Contabilidade, Universidade de São Paulo, São Paulo.

Murcia, F. D., \& Santos, A. (2009a). Regulação contábil e a divulgação de informações de operações com instrumentos financeiros derivativos: análise do impacto da CVM no 566/08 e da CVM no 475/08 no disclosure das companhias abertas no Brasil. Revista de Contabilidade e Organizações, 3(6), 3-21.

Murcia, F. D., \& Santos, A. (2009b). Fatores determinantes do nível de disclosure voluntário das companhias abertas no Brasil. Revista de Educação e Pesquisa em Contabilidade, 3(2), 72-95.

Murcia, F. D. R., Souza, M. M. D., Wuergues, A. F. E., \& Duarte, G. (2013). Realização ou estorno da reserva de reavaliação: fatores determinantes da escolha das companhias abertas no Brasil após o advento da Lei 11.638/07. ASAA-Advances in Scientific and Applied Accounting, 6(1), 112-133.

Nguyen, T. M. H., Nguyen, N. T., \& Nguyen, H. T. (2020). Factors affecting voluntary information disclosure on annual reports: listed companies in Ho Chi Minh City Stock Exchange. Journal of Asian Finance, Economics and Business, 7(3), 53-62.

Rufino, M. A., \& Monte, P. A. (2014). Fatores que explicam a divulgação voluntária das 100 empresas com ações mais negociadas na BM\&FBovespa. Sociedade, Contabilidade e Gestão, 9(3), 59-75.

Salotti, B. M., \& Yamamoto, M. M. (2008). Divulgação voluntária da demonstração dos fluxos de caixa no mercado de capitais brasileiro. Revista Contabilidade \& Finanças - USP, 19(48), 37-49.

Silva, T. L., Alberton, L., \& Vicente, E. F. R. (2013). Práticas de disclosure voluntário das empresas do setor elétrico: uma análise a partir do modelo de Murcia. Revista Catarinense da Ciência Contábil, 12(35), 48-62.

Silveira, A. D. M. (2010). Governança corporativa no Brasil e no mundo: teoria e prática. São Paulo: Elsevier.

Sousa, C. B., Silva, A. F., Ribeiro, M. S., \& Weffort, E. F. J. (2014). Valor de mercado e disclosure voluntário: estudo empírico em companhias listadas na BM\&FBOVESPA. Revista Ambiente Contábil, 6(2), 94-115.

Thoresson, A., \& Niléhn, P. (2014). Determinants of voluntary disclosure in Swedish corporate annual reports. Master Thesis, Uppsala University.

Verrecchia, R. E. (1983). Discretionary disclosure. Journal of Accounting and Economics, 5, 179-194.

Verrecchia, R. E. (2001). Essays on disclosure. Journal of Accounting and Economics, 32(1), 97-180.

Zagonov, M., Pettinicchio, A., \& Salganik-Shoshan, G. (2017). Audit quality, bank risks, and crosscountry regulations. Economics Bulletin, 37(3), 1666-1687.

Zaini, S. M., Sharma, U. P., \& Samkin, G. (2017). Impact of ownership structure on level of voluntary disclosure in annual reports: comparison between listed family-controlled and nonfamily-controlled companies in Malaysia. Australian Academy of Accounting and Finance Review, 3(3), 140-155. 